\title{
Homotopy Structure of 5d Vacua
}

\author{
Eun Kyung Park \\ The Liberal Arts Course, Kyungsung University, Pusan 608-736, Korea \\ and \\ Pyung Seong Kwon* \\ Department of Physics, Kyungsung University, Pusan 608-736, Korea
}

\begin{abstract}
It is shown that flat zero-energy solutions (vacua) of the 5d Kaluza-Klein theory admit a non-trivial homotopy structure generated by certain Kaluza-Klein excitations. These vacua consist of an infinite set of homotopically different spacetimes denoted by $\mathcal{M}_{5}^{(n)}$, among which $\mathcal{M}_{5}^{(0)}$ and $\mathcal{M}_{5}^{(1)}$ are especially identified as $M_{4} \times S^{1}$ and $M_{5}$, the vacuum states of the 5d Kaluza-Klein theory and the $5 \mathrm{~d}$ general relativity, respectively (where $M_{k}$ represents the $k$-dimensional Minkowski space).
\end{abstract}

PACS number : 04.50.+h

Keywords : 5d Kaluza-Klein, homotopy, massive excitation, 5d vacuum

${ }^{*}$ E-mail:bskwon@star.ks.ac.kr 
The 5d Kaluza-Klein theory is distinguished from the ordinary $5 \mathrm{~d}$ general relativity by the fact that the background vacuum is assumed to be the product $M_{4} \times S^{1}$ instead of $M_{5}$, where $M_{k}$ represents the $k$-dimensional Minkowski space. Having zero energy, the manifolds $M_{4} \times S^{1}$ and $M_{5}$ are both qualified for the vacuum state of the $5 \mathrm{~d}$ theory of gravity. Classically, there is no way to determine which is more appropriate than the other. The only basis for choosing $M_{4} \times S^{1}$ as the vacuum state of the 5 d Kaluza-Klein theory is that it includes the compactified dimension which is crucial in order to admit an internal gauge group $U(1)$ in the reduced theory. In fact, the compactification is an essential ingredient of any higher dimensional theory (including string theory) based on the Kaluza-Klein theory. The particle spectrum is then obtained by expanding around the vacuum $M_{4} \times S^{1}$; one finds a finite number of massless modes, and an infinite tower of massive (excitation) modes. In traditional theories the low-energy physics would be mostly governed by the dynamics of the massless modes alone, because the energy scale of the massive modes is about of order of the Planck scale. However, this is not to be the case anymore once we adopt the scenario that extra dimensions be very large. Recently, it has been suggested that the old hierarchy problem can be solved in the framework of higher-dimensional theories by taking extra dimensions to be very large 1]. In this scenario the energy scale of the massive modes (which is of order the inverse of the radius of $S^{1}$ ) could sufficiently lower down to the level of low energy physics, and one can imagine that the massive modes perhaps play an important role even in the low energy limit. In this paper, we examine the effect of the massive excitations on the geometry or topology of the background spacetime $M_{4} \times S^{1}$. Then we end up with a remarkable result that $5 \mathrm{~d}$ vacua admit a non-trivial homotopy structure ; $5 \mathrm{~d}$ vacua consist of an infinite set of homotopically different spacetimes denoted by $\mathcal{M}_{5}^{(n)}$, and where $\mathcal{M}_{5}^{(0)}$ and $\mathcal{M}_{5}^{(1)}$ are especially identified as $M_{4} \times S^{1}$ and $M_{5}$, respectively.

We start the discussion with a metric

$$
\begin{aligned}
d s^{2} & =-d t^{2}+d r^{2}+r^{2}\left(d \theta^{2}+\sin ^{2} \theta d \phi^{2}\right)+\Phi^{2}\left(x^{\alpha}, x^{5}\right)\left[d x^{5}+A_{\mu}\left(x^{\alpha}, x^{5}\right) d x^{\mu}\right]^{2} \\
& =\stackrel{4}{g}_{\mu \nu} d x^{\mu} d x^{\nu}+\Phi^{2}\left[d x^{5}+A_{\mu} d x^{\mu}\right]^{2} \\
& =\stackrel{4}{\eta}_{a b} \omega^{a} \omega^{b}+\left(\omega^{5}\right)^{2}, \quad(\mu, \nu, \alpha=0,1,2,3 ; a, b=0,1,2,3)
\end{aligned}
$$

where $\stackrel{4}{\eta}_{a b}$ is the 4 d flat Minkowski metric, and

$$
\omega^{a}=e_{\mu}^{a} d x^{\mu}, \quad \omega^{5}=\Phi\left(d x^{5}+A_{\mu} d x^{\mu}\right)
$$

are basis 1-forms of the orthonormal frame. From Eq.(1) we see that the fields $\Phi$ and $A_{\mu}$ have $x^{5}$-dependence, meaning that they include massive modes. In the reduced $4 \mathrm{~d}$ sector, $\Phi$ and $A_{\mu}$ are identified as the Brans-Dicke scalar and the $U(1)$ gauge potential, 
respectively. The vierbein $e^{a}{ }_{\mu}$, on the other hand, are functions of $x^{\alpha}$ alone ${ }^{1}$, so they commute with $\partial_{5}$. The non-vanishing components of Riemann tensor are then calculated in the orthonormal frame to give ${ }^{2}$

$$
\begin{aligned}
& \stackrel{5}{R}_{a b c d}=-\frac{1}{2}\left(\hat{f}_{a b} \hat{f}_{c d}+\hat{f}_{a}\left[c \hat{f}_{b} d\right]\right), \\
& \stackrel{5}{R}_{a b c 5}=\mathcal{D}_{[a} \hat{f}_{b] c}-F_{c} \hat{f}_{a b}, \\
& \stackrel{5}{R}_{a 5 b 5}=-\mathcal{D}_{(a} F_{b)}-\frac{1}{4} \hat{f}_{a c} \hat{f}_{b}^{c}-F_{a} F_{b},
\end{aligned}
$$

where $\hat{f}_{a b}$ is defined by $\hat{f}_{a b} \equiv \Phi f_{a b}$ and

$$
\begin{gathered}
f_{a b}=e_{a}{ }^{\mu} e_{b}{ }^{\nu}\left(D_{\mu} A_{\nu}-D_{\nu} A_{\mu}\right) \equiv e_{a}{ }^{\mu} e_{b}{ }^{\nu} f_{\mu \nu}, \quad\left(D_{\mu} \equiv \partial_{\mu}-A_{\mu} \partial_{5}\right), \\
F_{a}=e_{a}{ }^{\mu}\left(\partial_{\mu} \Phi-\partial_{5} \hat{A}_{\mu}\right) / \Phi \equiv e_{a}{ }^{\mu} F_{\mu}, \quad\left(\hat{A}_{\mu} \equiv \Phi A_{\mu}\right), \\
\mathcal{D}_{a}=e_{a}{ }^{\mu}\left(\nabla_{\mu}-A_{\mu} \partial_{5}\right) \equiv e_{a}{ }^{\mu} \mathcal{D}_{\mu},
\end{gathered}
$$

and in Eq.(8), $\nabla_{\mu}$ represents the ordinary covariant derivative associated with the metric $\stackrel{4}{g}_{\mu \nu}$. Also, $f_{\mu \nu}$ in Eq.(6) is a generalization of the Maxwell field strength ; it takes the same form as the conventional Maxwell field strength except that the ordinary derivative $\partial_{\mu}$ is replaced by the covariant derivative $D_{\mu}$. Without $x^{5}$-dependency the derivative $D_{\mu}$ reduces to $\partial_{\mu}$, and consequently $f_{\mu \nu}$ becomes the conventional Maxwell field strength.

Now we look for flat (vacuum) solutions which satisfy the equations $\stackrel{5}{R}$ abcd $=\stackrel{5}{R_{a b c 5}}=$ $\stackrel{5}{R}_{a 5 b 5}=0$. A set of the simplest solutions to these equations may be obtained by setting

$$
\begin{aligned}
& f_{\mu \nu}=D_{\mu} A_{\nu}-D_{\nu} A_{\mu}=0, \\
& F_{\mu}=\frac{1}{\Phi}\left(\partial_{\mu} \Phi-\partial_{5} \hat{A}_{\mu}\right)=0,
\end{aligned}
$$

\footnotetext{
${ }^{1}$ The $x^{5}$-independency of $e^{a}{ }_{\mu}$ greatly simplifies the calculation. See ref. [2]

${ }^{2}$ Note that the constraints $\mathcal{D}_{[a} \hat{f}_{b c]}-F_{[a} \hat{f}_{b c]}=0$ and $\mathcal{D}_{[a} F_{b]}+\left(\partial_{5} \hat{f}_{a b}\right) / 2 \Phi=0$ are required by the symmetry $\stackrel{5}{R}_{A B C D}=\stackrel{5}{R}_{C D A B}$, and they have been used to obtain $\stackrel{5}{R}_{a b c 5}$ and $\stackrel{5}{R}_{a 5 b 5}$. Also, note that the $4 \mathrm{~d}$ sector of the spacetime in Eq.(1) is flat, so the $4 \mathrm{~d}$ Riemann tensor $\stackrel{4}{R_{a b c d}}$ vanishes and does not appear in Eq.(3). The Riemann tensors in Eqs.(3) to (5) are in fact a generalization of those in ref. 3].

${ }^{3}$ The derivative $D_{\mu}$ (more generally $\mathcal{D}_{\mu}$ ), acting on a tensor, leaves its components invariant under the gauge transformation: $x^{5} \rightarrow x^{5^{\prime}}=x^{5}+f\left(x^{\alpha}\right), A_{\mu} \rightarrow A_{\mu}^{\prime}=A_{\mu}-\partial_{\mu} f$. See ref. [4] for this. Thus we see that the Eqs.(3), (4) and (5) are all expressed in gauge-covariant form.
} 
and in particular Eq.(9) is immediately solved by an ansatz

$$
A_{t}=A_{\theta}=A_{\phi}=0, \quad A_{r}=A_{r}\left(r, x^{5}\right), \quad \Phi=\Phi\left(r, x^{5}\right) .
$$

Equation (11) is actually the most general ansatz preserving spherical symmetry, and $A_{\mu}$ in Eq.(11) may be regarded as a pure gauge in the sense that it gives $f_{\mu \nu}=0$. With this ansatz, the metric in Eq.(1) can be recast into the form

$$
d s^{2}=-d t^{2}+d \hat{r}^{2}+r^{2}\left(d \theta^{2}+\sin ^{2} \theta d \phi^{2}\right)+\frac{\Phi^{2}}{1+\hat{A}_{r}^{2}}\left(d x^{5}\right)^{2}, \quad\left(\hat{A}_{r} \equiv \Phi A_{r}\right),
$$

where $d \hat{r}$ is defined by

$$
d \hat{r}=\left(1+\hat{A}_{r}^{2}\right)^{1 / 2} d r+\frac{\Phi \hat{A}_{r}}{\left(1+\hat{A}_{r}^{2}\right)^{1 / 2}} d x^{5} .
$$

Equation (13) indicates that the variable $\hat{r}$ is a function of $r$ and $x^{5}$; i.e., $\hat{r}=f\left(r, x^{5}\right)$ with

$$
\frac{\partial f}{\partial r}=\left(1+\hat{A}_{r}^{2}\right)^{1 / 2}, \quad \frac{\partial f}{\partial x^{5}}=\frac{\Phi \hat{A}_{r}}{\left(1+\hat{A}_{r}^{2}\right)^{1 / 2}} .
$$

With the aid of Eq.(10) one then finds from the equations in (14) that the condition $\partial^{2} f / \partial r \partial x^{5}=\partial^{2} f / \partial x^{5} \partial r$ implies that $\partial_{r} \hat{A}_{r}=0$; i.e., $\hat{A}_{r}$ must be a function of $x^{5}$ alone:

$$
\hat{A}_{r} \equiv Y\left(x^{5}\right)
$$

Thus the equations in (14) are now integrated to give

$$
\begin{gathered}
f=\hat{r}=r\left(1+Y^{2}\right)^{1 / 2}+g\left(x^{5}\right), \\
\Phi=r Y^{\prime}+\frac{\left(1+Y^{2}\right)^{1 / 2}}{Y} g^{\prime},
\end{gathered}
$$

where $g\left(x^{5}\right)$, which has been introduced as an integral constant, is an arbitrary function of $x^{5}$ alone, and the 'prime' in Eq.(17) denotes the $x^{5}$-derivative. Note that $\hat{A}_{r}$ and $\Phi$ in Eqs.(15) and (17) indeed describe the flat solution satisfying $\stackrel{5}{R_{A B C D}}=0$; one can readily check that they satisfy Eq.(10). Now we impose the condition

$$
\lim _{Y, Y^{\prime} \rightarrow 0} \Phi=\text { constant } \equiv \Phi_{0}
$$

which suggests that the solution we are to find is the one that reduces to the KaluzaKlein vacuum $M_{4} \times S^{1}$ as $A_{\mu} \rightarrow 0$; note that $M_{4} \times S^{1}$ with $A_{\mu}=0$ and $\Phi=$ constant is also a solution to Eq.(10). The condition in Eq.(18) immediately implies that

$$
g^{\prime}=\Phi_{0} Y
$$


and therefore $\Phi$ in Eq.(17) becomes

$$
\Phi=r Y^{\prime}+\Phi_{0}\left(1+Y^{2}\right)^{1 / 2}
$$

Using all this, one can show that the metric in Eq.(12) can be converted into the form

$$
d s^{2}=-d t^{2}+\left(1-\frac{\Phi_{0}^{2} Y^{2}}{R^{2}}\right) d \rho^{2}+\frac{\rho^{2}}{1+Y^{2}}\left(d \theta^{2}+\sin ^{2} \theta d \phi^{2}\right)+R^{2}\left(d x^{5}+\frac{\Phi_{0} Y}{R^{2}} d \rho\right)^{2},
$$

where $\rho$ and $R$ are defined by

$$
\begin{gathered}
\rho=\hat{r}-g=r\left(1+Y^{2}\right)^{1 / 2}, \\
R=\left[\Phi_{0}^{2} Y^{2}+\left(\rho \frac{Y^{\prime}}{1+Y^{2}}+\Phi_{0}\right)^{2}\right]^{1 / 2} .
\end{gathered}
$$

So far, the function $Y\left(x^{5}\right)$ and the constant $\Phi_{0}$ have been entirely arbitrary except that they should satisfy the condition (18). Now let us take

$$
Y_{n}\left(x^{5}\right)=\tan \frac{n x^{5}}{2 R_{c}}, \quad \Phi_{0}=\delta_{n 0}, \quad(n=0,1,2, \cdots)
$$

where $R_{c}$ represents the compactification radius of the fifth-dimension (note that Eq.(24) respects the condition (18) as $n \rightarrow 0$ ). By Eqs.(15), (20) and (24), the gauge field $A_{r}$ becomes

$$
A_{r}\left(r, x^{5}\right)=a_{n}(r) \sin \frac{n x^{5}}{R_{c}}
$$

with

$$
a_{n}(r)=\frac{R_{c}}{n r+2 R_{c} \delta_{n 0}},
$$

which shows that taking $Y_{n}\left(x^{5}\right)$ as in Eq.(24) implies that we are considering a situation where the n-th excitation of the gauge field (together with the scalar field induced by this gauge field) is present in the background spacetime $M_{4} \times S^{1}$ (see Eq.(1)). In this case the field $\Phi\left(r, x^{5}\right)$ takes the form

$$
\Phi\left(r, x^{5}\right)=\frac{n r}{2 R_{c}} \sec ^{2} \frac{n x^{5}}{2 R_{c}}+\delta_{n 0},
$$

and we see that $A_{r} \rightarrow 0, \Phi \rightarrow 1$ for $n=0$. Namely, the $n=0$ state simply describes the compactified vacuum $M_{4} \times S^{1}$ without any gauge, or scalar field. For $n \neq 0$, on the other hand, both fields are present in the form of $A_{r} \sim 1 / r$ and $\Phi \sim r$, respectively (the presence of the gauge excitation necessarily demands the presence of the scalar field with a behavior of $\Phi \sim r$, as can be checked from Eq.(10)). Then, what happens to the spacetime by the presence of these excitations $\Phi$ and $A_{r}$ ? By Eq.(24), the metric (21) simplifies to

$$
d s^{2}=-d t^{2}+d \rho^{2}+\rho^{2} \sin ^{2} \chi_{n}\left(d \theta^{2}+\sin ^{2} \theta d \phi^{2}\right)+\left(\rho d \chi_{n}+\delta_{n 0} d x^{5}\right)^{2}
$$


with $\chi_{n}$ defined by

$$
\chi_{n}\left(x^{5}\right)=\frac{n x^{5}}{2 R_{c}}+\frac{\pi}{2}
$$

which, after all, implies that the metric (28) is equivalent under (24) to the metric in Eq.(1) with $\Phi$ and $A_{\mu}$ given by Eqs.(25) and (27). Indeed, for $n=0$, it reduces to the flat $M_{4} \times S^{1}$ :

$$
d s_{(0)}^{2}=-d t^{2}+d \rho^{2}+\rho^{2}\left(d \theta^{2}+\sin ^{2} \theta d \phi^{2}\right)+\left(d x^{5}\right)^{2},
$$

which is just the metric in Eq.(1) with $A_{\mu}=0, \Phi=1$. For $n \neq 0$, on the other hand, the metric (28) becomes

$$
d s_{(n)}^{2}=-d t^{2}+d \rho^{2}+\rho^{2}\left[d \chi_{n}^{2}+\sin ^{2} \chi_{n}\left(d \theta^{2}+\sin ^{2} \theta d \phi^{2}\right)\right]
$$

the $5 \mathrm{~d}$ flat Minkowski metric! The topology of spacetime has been changed by the non-zero excitation modes. The toroidal compactification $M_{4} \times S^{1}$ in Eq.(1) has been converted into the non-compact spacetime described by the metric in Eq.(31). Furthermore, let us change the variable $x^{5} \rightarrow y_{n}$ by the equation

$$
y_{n}=x^{5}+\frac{\pi R_{c}}{n} \text {. }
$$

Under Eq.(32), the metric (1) (with $A_{r}$ and $\Phi$ given by Eqs.(25) and (27)) takes the same form as before : i.e.,

$$
d s^{2}=-d t^{2}+d r^{2}+r^{2}\left(d \theta^{2}+\sin ^{2} \theta d \phi^{2}\right)+\Phi^{2}\left(r, y_{n}\right)\left[d y_{n}+A_{r}\left(r, y_{n}\right) d r\right]^{2}
$$

with $^{4}$

$$
A_{r}\left(r, y_{n}\right)=-a_{n}(r) \sin \frac{n y_{n}}{R_{c}}, \quad \Phi\left(r, y_{n}\right)=\frac{n r}{2 R_{c}} \csc ^{2} \frac{n y_{n}}{2 R_{c}}+\delta_{n 0},
$$

but $\chi_{n}$ in (31) is now written (from (29)) as

$$
\chi_{n}(y)=\frac{n y_{n}}{2 R_{c}}, \quad\left(0 \leq y_{n} \leq 2 \pi R_{c}\right)
$$

This is remarkable. Equation (35) suggests that the $5 \mathrm{~d}$ vacua admit a non-trivial homotopy structure. Note that the angle $\chi_{n}$ varies from 0 to $n \pi$ as $y_{n}$ makes a single turn from 0 to $2 \pi R_{c}$ around $S^{1}$, which in turn means that the set of variables $\left(\chi_{n}, \theta, \phi\right)$ in (31) covers the hypersurface $S^{3} n$ times when $\left(y_{n}, \theta, \phi\right)$ in (33) covers $S^{2} \times S^{1}$ just once. The $3 \mathrm{~d}$ manifold described by $\left(\chi_{n}, \theta, \phi\right)$ can be regarded as a 3-loop $\alpha_{n}\left(t_{1}, t_{2}, t_{3}\right)$, where $t_{i}$, the coordinates of $3 \mathrm{~d}$ cube $I_{3}$, are defined by the map : $t_{1}=y_{n} / 2 \pi R_{c}, t_{2}=\theta / \pi$

\footnotetext{
${ }^{4}$ The metric (33) seems to have a singularity at $y_{n}=0$ (or $2 \pi R_{c}$ ) because $\Phi\left(r, y_{n}\right)$ in (34) is singular there. However, this singularity is obviously artificial ; notice that the metric (33) is a flat solution satisfying $R_{A B C D}=0$ in orthonormal frame.
} 
and $t_{3}=\phi / 2 \pi$, which is an homeomorphism $f: S^{2} \times S^{1} \longrightarrow I_{3}$. A collection of these 3 loops which cover $S^{3} n$ times constitutes the $n$-th equivalence class of the 3 rd. homotopy group $\pi_{\mathbf{3}}\left(S^{3}\right)$. The spatial subsector of the spacetime described by Eq.(31) is essentially a pile of such 3-loops. This suggests that the spacetime described by Eq.(31) (let us call it $\mathcal{M}_{5}^{(n)}$ ) belongs to the $n \neq 0$ homotopy class of $\pi_{\mathbf{3}}\left(S^{3}\right)$, though $M_{4} \times S^{1}$ with no excitations especially belongs to the $n=0$ class since it is obtained by simply taking ${ }^{5}$ $n=0$. Further, for $n=1$, it is obvious from Eq.(35) that $\mathcal{M}_{5}^{(n)}$ is precisely identified as the ordinary $5 \mathrm{~d}$ Minkowski space $M_{5}$. But note that, in general, $\mathcal{M}_{5}^{(n)}$ is not $M_{5}$ itself $; \mathcal{M}_{5}^{(n)}$ is an $n$-fold cover of $M_{5}$. Namely, it is a fiber bundle over $M_{5}$ with fiber $F$ a discrete set of $n$ points. In short, $5 \mathrm{~d}$ vacua consist of an infinite set of homotopically different spacetimes $\mathcal{M}_{5}^{(n)}$, among which the cases of $n=0$ and $n=1$ are especially identified as the background vacua of the $5 \mathrm{~d}$ Kaluza-Klein theory and the $5 \mathrm{~d}$ general relativity, respectively ; i.e., $M_{4} \times S^{1}=\mathcal{M}_{5}^{(0)}$, and $M_{5}=\mathcal{M}_{5}^{(1)}$. Such a homotopy structure manifests itself once there is a defect, or a point particle at $\rho=0$. With a defect or point particle at $\rho=0$, the spatial subsector of $M_{5}$ is not simply connected, and the 3 -loops which contain $\rho=0$ are not shrinkable. So each $\mathcal{M}_{5}^{(n)}$, a pile of such 3-loops which enclose the point $\rho=0 n$ times, belongs to a different homotopy class.

Though the above discussion has its own right in 5d Kaluza-Klein theories, it may be applied to any other higher dimensional theories with toroidal compactification. For instance, in the eleven-dimensional theory compactified on $X \times S^{1} / Z_{2}$ (or equivalently, in the strong coupling limit of the $E_{8} \times E_{8}$ heterotic string) it is believed that the radius of the orbifold $S^{1}$ is larger than the volume of the Calabi-Yau manifold $X$, and there is a regime where our spacetime appears five-dimensional [5, 6]. To the lowest order of the $11 \mathrm{~d}$ Newton constant $\kappa$, the ( $5 \mathrm{~d}$ sector of the) ground state metric takes the form of the 5 d Kaluza-Klein vacuum $M_{4} \times S^{1}$. So in this case the vacua of the theory could admit the homotopy structure under discussion. If this is the case, it then follows that the T-duality could break down due to the presence of the excitations. Note that for $n \neq 0$ the compactified dimension $S^{1}$ disappears due to the excitation modes ; the map $f$ : $S^{2} \times S^{1} \rightarrow S^{3}$ takes $S^{1}$ to the great circle of $S^{3}$. Thus the winding number (of the closed string) is not a topological number here and consequently the term corresponding to winding modes does not exist in the mass formula, and it leads to the conjecture that the T-duality might break down in the presence of excitations.

So far, we have considered only the (excitations of the) Kaluza-Klein components (i.e., $5 \mathrm{~d}$ metric components) as the source of the topology change of the spacetime.

\footnotetext{
${ }^{5}$ Eq.(35) (i.e., the variable change $x^{5} \rightarrow y_{n}$ in Eq.(32)) is in fact applicable to the $n=0$ case either. Note that since $\lim _{n \rightarrow 0} n y_{n}=\pi R_{c}$ from (32), we see that the metric (33) reduces to the flat $M_{4} \times S^{1}$ as $n \rightarrow 0$. This is the same result that we obtain from the metric (1) with $A_{r}$ and $\Phi$ given by Eqs.(25) and (27).
} 
But in the 5d bulk spacetime there also exist other fields besides the Kaluza-Klein components $[6]$. For instance, we may consider the case where a $5 \mathrm{~d} U(1)$ gauge field $^{6}$ $\mathcal{A}_{\mu}$ exists in the bulk spacetime, with field strength $\mathcal{F}_{\mu \nu}$ (see for instance ref.[7]), and it takes the place of the Kaluza-Klein vector $A_{\mu}$. Indeed, disregarding the components $\mathcal{F}_{\mu 5}$, one finds that the $4 \mathrm{~d}$ effective action for $\mathcal{A}_{\mu}$ takes the same form as the action for $A_{\mu}$, which suggests that the homotopy structure of the $5 \mathrm{~d}$ vacua can be generated even by (the excitations of) bulk fields, rather than Kaluza-Klein components.

The result of this paper is quite analogous to the case of the $\theta$-vacua of the Yang-Mill theory. In both cases the vacua admit the same homotopy structure with an infinite set of homotopy classes each of which is characterized by an integer $n$. In the Yang-Mill theory the integer $n$ is identified with the Pontryagin index $q \sim \operatorname{Tr} \int d^{4} x F^{\mu \nu} \widetilde{F}_{\mu \nu}$, and the vacua belonging to different homotopy classes are connected by a Euclidean (instanton) solution. In the present paper the integer $n$ is simply an excitation number of the pure gauge $A_{\mu}$, and the instanton solution interconnecting two different vacua does not exist here. But notice that in our case there exist gauge transformations which mix the massive and massless modes [8] of $A_{\mu}$, and consequently the spacetimes belonging to different classes can be mixed by such gauge transformations. The gauge transformation which mixes the massive and massless modes can be generated by allowing gauge parameter $\xi$ to depend on $x^{5}$. For instance, the transformation which mixes the $n$-th and $(n-k)$-th modes (in the Fourier exponential series) of $A_{\mu}$ takes the form (see ref. [8])

$$
\delta_{k} A_{\mu n}=\delta_{n k} \partial_{\mu} \xi_{k}+i(n-2 k) \xi_{k} A_{\mu(n-k)} / R_{c}
$$

where $A_{\mu n}$ and $\xi_{n}$ are the $n$-th components of the Fourier exponential series

$$
\begin{aligned}
A_{\mu}\left(x^{\alpha}, x^{5}\right) & =\sum_{n} A_{\mu n}\left(x^{\alpha}\right) e^{i n x^{5} / R_{c}}, \\
\xi\left(x^{\alpha}, x^{5}\right) & =\sum_{n} \xi_{n}\left(x^{\alpha}\right) e^{i n x^{5} / R_{c}},
\end{aligned}
$$

respectively, and $\delta_{k}$ represents the transformation induced by $\xi_{k}$. Since $\mathcal{M}_{5}^{(n)}$ is equivalent to $\mathcal{M}_{5}^{(0)}$ plus the $n$-th excitation (in the Fourier sine-series) of $A_{\mu}$, the transformation (36) apparently mixes $\mathcal{M}_{5}^{(n)}$ with other spacetimes belonging to different classes. This is quite remarkable. Note that a gauge transformation essentially does not change the physics of a system. But the above argument suggests that $\mathcal{M}_{5}^{(n)}$ is not invariant under a 'large' gauge transformation of the form (36), which immediately leads us to suspect that spacetimes $\mathcal{M}_{5}^{(n)}$ (most importantly $\mathcal{M}_{5}^{(0)}=M_{4} \times S^{1}$ and $\mathcal{M}_{5}^{(1)}=M_{5}$ ) may not be the physical states of the theory. Namely, the suggested conjecture is that

\footnotetext{
${ }^{6}$ Or it could be the remnant of dimensionally reduced higher-rank tensor fields.
} 
$M_{4} \times S^{1}$ may not be the true physical vacuum of the $5 \mathrm{~d}$ Kaluza-Klein theory ; it serves as a vacuum only when we do not consider the 'large' gauge transformation. The physical vacuum of the $5 \mathrm{~d}$ Kaluza-Klein theory may perhaps be a superposition of an infinite number of $\mathcal{M}_{5}^{(n)}$, analogously to the case of the $\theta$-vacua of the Yang-Mill theory.

This research was supported by Kyungsung University research grants in 2003.

\section{References}

[1] N. Arkani-Hamed, S. Dimopoulos and G. Dvali, Phys. Lett. B429, 263 (1998) ; I. Antoniadis, N. Arkani-Hamed, S. Dimopoulos, and G. Dvali, Phys. Lett. B436, 257 (1998) ; N. Arkani-Hamed, S. Dimopoulos, and G. Dvali, Phys. Rev. D59, 086004 (1999).

[2] P. S. Wesson and J. P. Leon, J. Math. Phys. 33, 3883 (1992) ; 34, 4080 (1993).

[3] Y. Thiry, C. R. Acad. Sci. (PARIS) 226, 216 (1948).

[4] A. Einstein and P. Bergmann, Ann. Math. 39, 683 (1938).

[5] E. Witten, Nucl. Phys. B417, 135 (1996) ; T. Banks and M. Dine, Nucl. Phys. B479, 173 (1996).

[6] A. Lukas, B. A. Ovrut, K.S. Stelle and D. Waldram, Phys. Rev. D59, 086001 (1999) ; Nucl. Phys. B552, 246 (1999).

[7] R. Sundrum, Phys. Rev. D59, 085010 (1999).

[8] L. Dolan and M. J. Duff, Phys. Rev. Lett. 52, 14 (1984). 